\title{
Innovative Wireless Sensors Networks View based on Data Fusion Optimization
}

\section{Wen Tsai Sung*}

Department of Electrical Engineering, National Chin-Yi University of Technology, Zhongshan Rd, Taiping, Taichung 41170, Taiwan

Wireless sensors networks is a rapidly developing area, a combination of network, mathematics and computing technology, in order to enhance the complex sensors network and data aggregation. Traditional Data Fusion method does not have the ability to process hung amounts sensors signals that is why the wireless sensors networks design often only one cluster or one layer framework. These innovative wireless sensors networks technology brings together some of the optimal fusion of innovative information technology and methods and it provides to the readers on this issue have further improved data fusion in wireless sensors networks. These innovative approaches contain the following topics: The application of general systems theory; evolution-computing application; artificial intelligence application; Innovative optimization algorithm application; optimization sensor big-data analysis and application; interactive mobile monitoring and measurement; distributed deployment strategies and techniques application; Arduino circuit board or other microcontrollers and multi-sensors application; mobile real-time optimization etc.

Today's mobile devices or multi-sensors exhibit excellent performance and very soon some mobile device functions will gradually replace the personal computer. Mobile devices include smart phones, PDAs, tablet PCs and thin notebooks. Multi-sensors are set by a variety of different forms and types of sensors consisting of sensor network system. Using smart phones, tablet PCs or multi-sensors to perform real-time remote monitoring is very efficient. The operator is usually performing only real-time monitoring while other optimization analysis is still performed using computers. When we use computers to optimize the analysis it is usually no longer real-time analysis. Therefore, this above issues proposes smart devices for instant realtime data optimization. When we optimize data via mobile devices, we can monitor on-site interactive systems in real-time and perform remote optimization adjustments. This is a very efficient, innovative method for immediate adjustment action.

In the past we focused only on remote environment monitoring, with less on how to control or change the status quo. This above issues employed mobile devices to optimize remote sensing data and adjust the device to meet the remote environment optimized state. Mobile device optimization presents very good system interaction. For various types of sensor, front optimization of action will have different rendering techniques. For example, analog measurement sensors, the data returned can be done on the level of analysis and optimization. On the other hand, digital-type sensor, it is clear that we can get the polarized results. If this is obviously a high state and low state is used in the front-end analysis, it can create another result of the indication. Depending on the sensors measurement, we hope to come up with the corresponding analysis and optimization methods, the remote measurement results are better and do detail rendering of depth.

Wireless sensors networks involve technologies from three related areas: sensing, communication and computation (hardware, software, and algorithm). Lately a lot of research work has been done in all of these fields to make sensor nodes more intelligent and useful. Wireless sensors networks have emerged as a new information gathering paradigm based on the collaborative effort of a large number of sensing nodes. These above issues will allow scientists to develop smarter process strategies for multi-sensors signals and data. General optimization technology for solving or seeking convergence method to verify the existence or stability of wireless sensors networks, innovative wireless sensors networks research topics with the best solutions, through innovative optimization theory to the modeling and simulation of wireless sensors networks. Neural-computing, artificial intelligence, particle swarm optimization and genetic algorithm methods can be used to achieve and to verify the wireless sensors networks system combinational learning with interdisciplinary data fusion integration and sharing of resources. The development of this theme will be able to make more data fusion problems and research through the innovative optimization theory research methods and technology acquire a better advancement.

*Corresponding author: Wen Tsai Sung, Department of Electrical Engineering National Chin-Yi University of Technology, Zhongshan Rd, Taiping, Taichung 41170, Taiwan, Tel: +886-4-23924505-2150; Fax: +886-4-23924419; E-mail: songchen@ncut.edu.tw

Received October 24, 2013; Accepted October 25, 2013; Published October 26 2013

Citation: Sung WT (2013) Innovative Wireless Sensors Networks View based on Data Fusion Optimization. Sensor Netw Data Commun 1: 110. doi: 10.4172/2090 4886.1000110

Copyright: ( 2013 Sung WT. This is an open-access article distributed under the terms of the Creative Commons Attribution License, which permits unrestricted use, distribution, and reproduction in any medium, provided the original author and source are credited. 